\title{
Review
}

Mehran Alavi' ${ }^{1}$ Mehrdad Hamidi ${ }^{2,3}$

\section{Passive and active targeting in cancer therapy by liposomes and lipid nanoparticles}

\author{
${ }^{1}$ Department of Nanobiotechnology, Faculty of Science, Razi University, Kermanshah, Iran, E-mail: mehranbio83@gmail.com \\ ${ }^{2}$ Zanjan Pharmaceutical Nanotechnology Research Center (ZPNRC), Zanjan, Iran, E-mail: hamidim@zums.ac.ir \\ ${ }^{3}$ Department of Pharmaceutical Nanotechnology, School of Pharmacy, Zanjan University of Medical Sciences, Zanjan, Iran, \\ E-mail: hamidim@zums.ac.ir
}

\begin{abstract}
:
Considerable development in the application of injectable drug delivery systems for cancer therapy has occurred in the last few decades. These improvements include liposomes, lipid nanoparticles (LNPs), and other nanoparticles with or without macromolecular conjugates. For example, liposomal doxorubicin modified by poly(ethylene glycol) (Doxil) was the first liposome with anti-cancer effects which was approved by the US Food and Drug Administration, whereas Abraxane (modified albumin nanoparticles loaded by paclitaxel) was recently confirmed for the treatment of breast cancer. Recently, drug delivery systems by LNPs are an emerging technology with numerous advantages over conventional liposomes and chemotherapy using free drug treatment of cancer. These properties are biocompatibility, controlled and sustained release of anti-tumor drugs, and lower toxicity. Valuable experiments on these drug delivery systems offer better treatment of multidrugresistant cancers and lower cardiotoxicity. LNPs have been presented with high functionality in chemotherapeutic targeting of breast and prostate cancer. The basis for this targeting behavior has been shown to be both passive and active targeting. The main objective of this review was an overview of the current position of the liposome-based drug delivery systems in targeted anticancer chemotherapy.
\end{abstract}

Keywords: active targeting, cancer therapy, lipid nanoparticles, liposomes, passive targeting

DOI: $10.1515 / \mathrm{dmpt}-2018-0032$

Received: September 12, 2018; Accepted: November 20, 2018

\section{Introduction}

Significant advances in the application of injectable drug delivery systems for cancer therapy have occurred in the last decades. These advancements include the use of liposomes and other nanoparticles as macromolecular conjugates. For instance, liposomal doxorubicin modified by poly (ethylene glycol) (PEG) (Doxil) was the first liposome with anti-cancer effects approved by the US Food and Drug Administration (FDA), whereas Abraxane [paclitaxel (PTX) loaded in albumin nanoparticles] was recently produced for the treatment of breast cancer. There are several challenges related to modification of liposomes and their effects on cancer tissues [1], [2], [3], [4], [5], [6], [7], [8], [9]. As a typical class of nanomedicines, liposomes are nowadays prepared at the nano size range using different methods. In the nanomedicine field, the design of the nano-scaled devices and their interactions with cellular targets at the nano-scale are rapidly evolving. In this way, liposomes had been the first generation of nano-scale drug delivery systems approved for treatment of cancer (e.g. Doxil) and fungal infections (e.g. Ambisome) [10], [11], [12], [13], [14], [15]. Liposomes have an aqueous interior part, surrounded by one or more concentric bilayers of phospholipid structure. The diameter of liposomes is variable ranging from $1 \mathrm{~nm}$ to several microns. In the case of injectable clinical utilization, all liposome formulations are in the submicron ultra-filterable range of less than $200 \mathrm{~nm}$ size and can be considered as nanostructure systems. When amphiphilic lipids such as phospholipids are dispersed in water, liposomes are spontaneously formed. These structures are physically stable, and unlike polymeric particles, they are not covalently bound. Depending on the water solubility properties of the drug, it can be encapsulated in the aqueous core or in surrounding bilayer of the liposome [16], [17], [18]. Hydrophobic drugs are incorporated into the lipid membrane, whereas hydrophilic drugs are encapsulated within the central aqueous core [19], [20], [21], [22], [23], [24], [25]. Cancer therapy may benefit from nanocarriers via two major approaches including passive and active targeting with 
liposomes being potentially useful in both cases. In this review, we attempt to present practical information regarding recent advances with respect to injectable liposomes and lipid nanoparticle (LNP)-based drug delivery systems with passive and active targeting abilities for cancer therapy.

\section{Cancer disease and therapy hindrances}

Abnormal growth (neoplasm) of a tissue to produce an abnormal population of cells is referred to as tumor, which can be found in two major forms: benign or malignant. Cancer is the malignant form of neoplasm resulting in cells without a normal morphology and/or function [26]. These types of tissues have subdivisions of cells, interstitial and vascular. In the case of cellular type, cancers may be carcinoma, sarcoma, lymphoma, germ cells (pluripotent cells) tumors, and blastoma. This classification is based on the tissue of origin of the malignancy (Figure 1). Also, based on size and shape, cancer cells such as carcinoma type may be divided into small-cell, spindle cell, and giant cell carcinoma [27].

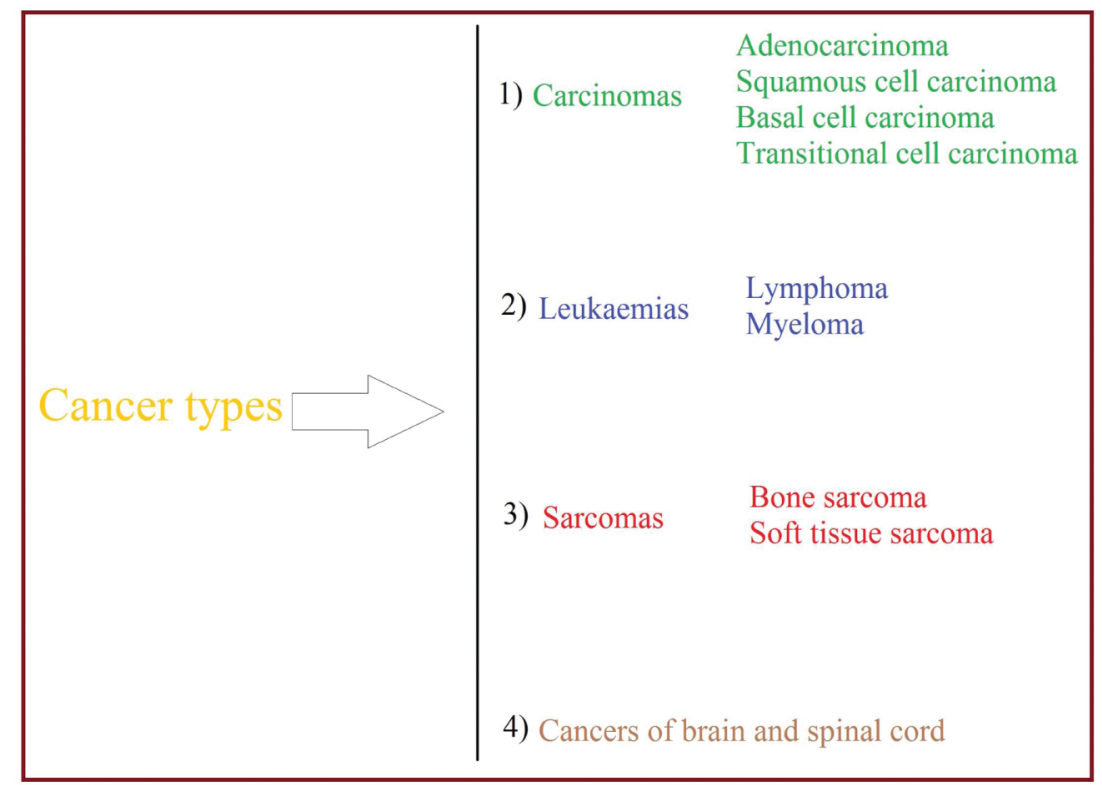

Figure 1: Cancer classification based on tissue types [26] and [27].

As illustrated in Figure 2, cancer therapy may be carried out by several approaches including chemotherapy, biological therapy, immunotherapy, hormone therapy, radiotherapy, stem cell transplant, using therapeutic vaccines, and surgery [28], [29], [30], [31], [32]. In chemotherapy, cancer cells are often destroyed or treated by cytotoxic and genotoxic drugs. This type of therapy has several major side effects such as nausea, fatigue, diarrhea, hair loss, disruption of mouth, pharynx mucosa, and bone marrow [33], [34]. In biological therapy, living organisms (mainly viruses and bacteria) or components of living organisms are utilized to treat cancer. Among viruses, anticancer properties of the mumps virus, Newcastle disease virus, reovirus, adenovirus, vaccinia virus, and the measles virus are approved by the US FDA [35]. Also, vaccines of bacillus Calmette-Guérin, weakened form of the tuberculosis bacteria, have been applied against bladder cancer [36]. Immunotherapy is one subtype of biological therapy. Application of monoclonal antibodies, adoptive cell transfer, cytokines, and vaccines in cancer treatment are located in the immunotherapy group [37]. In this way, the effect of BATF3dependent dendritic cells in improvement of antitumor efficiency by anti-PD-1 and anti-CD137 monoclonal antibodies against several types of cancer is approved [38]. The side effects of immunotherapy are pain, soreness, redness, swelling, rash, itchiness, and flu-like symptoms. 


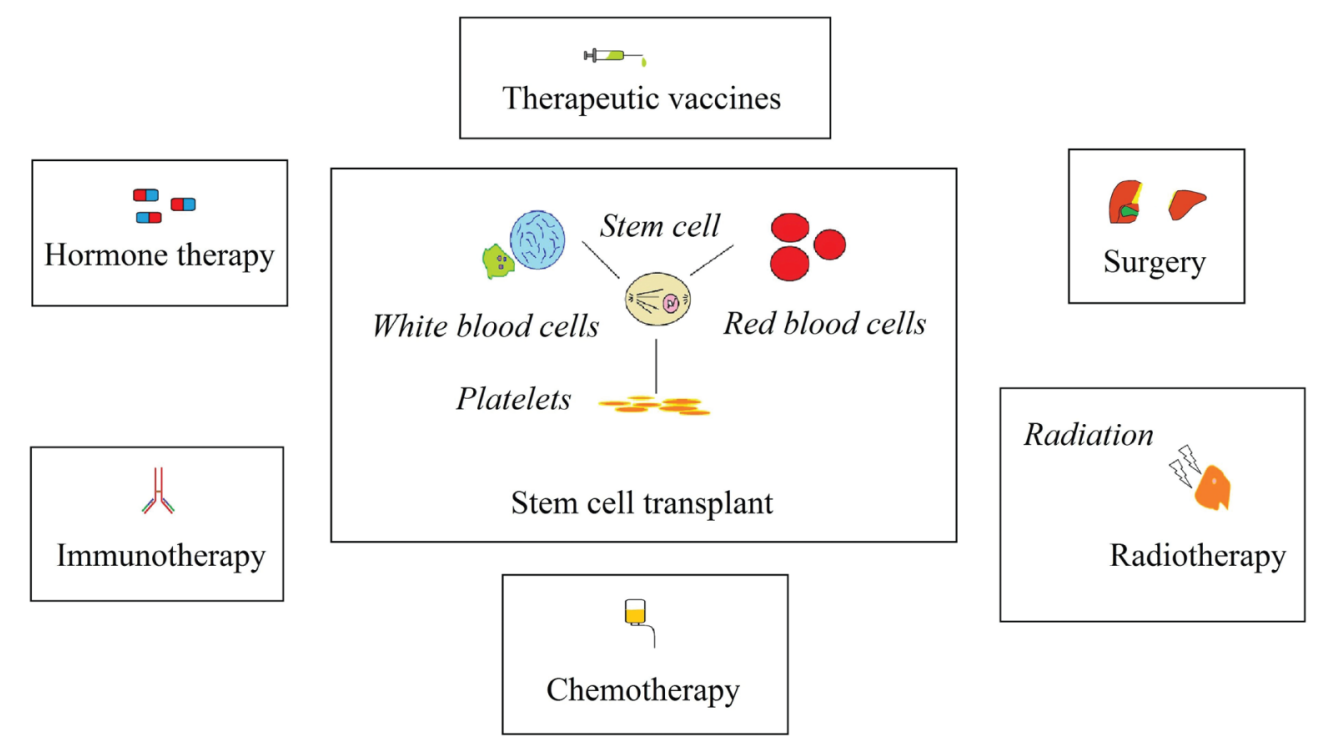

Figure 2: Diagram of cancer therapy approaches [28], [29], [30], [31], and [32].

Tumor receptors are a potential target for specific ligands or antibodies with or without delivery of a cytotoxic drug cargo. The pathophysiology of tumor neovasculature and the interaction of tumors with the stroma have a major role in tumor development. In fact, cancer is a disease caused by somatic mutations that result in the transformation of normal cells into malignant tumor cells. There are four major stages to phenotype progress of tumor cells: (1) appearance of abnormal cells, (2) high proliferation of tumor cells, (3) invasion to the surrounding tissues through angiogenesis, and (4) metastases. In stage 4 of cancer, there is abnormal migration of tumor cells from the primary tumor site through blood vessels or lymphatics to distant organs and formation of secondary tumors [39].

Competition for drug uptake by liver and kidneys, binding of protein with drug inactivation, glomerular filtration and urinary excretion of low molecular weight drugs, and low stability of drug in fluids (e.g. opening of the lactone ring of camptothecin analogs) are physiologic parameters that can seriously limit the drug distribution efficiency from plasma to tumors and neutralize their effects.

\section{Liposomes and LNPs}

\section{Liposomes}

Being one of the oldest while still promising drug carriers, liposomes are spherical structures made of a hydrophilic core surrounded by a bilayer made of some amphiphic lipid materials, mainly phospholipids. Generally, the size of liposomes can be in the range of $25 \mathrm{~nm}-2.5 \mu \mathrm{m}$ with one or several bilayer membranes [40]. Classification of liposomes is based on these two parameters, i.e. number of bilayers and size. In the case of bilayers number, there are unilamellar (with one phospholipid bilayer) and multilamellar (with several bilayers like an onion shape) liposomes with unilamellar type divided into two subtypes of small and large unilamellar liposomes (Figure 3) [42]. These structures are important in encapsulating of various drugs with different size, shape, and solubility in water. By modification of these vesicles, it may be possible to target specific organs, tissues, and cells [43], [44]. Also, based on the charge of the lipid constituents in the formation of liposomes, there are cationic and anionic liposomes. For example, cationic unilamellar liposomes can be synthesized by $\mathrm{N}$-[1-(2,3-dioleyloxy)propyl]-N,N,N-trimethylammonium chloride to deliver nucleic acids including miRNA, DNA, and siRNA to cells of interest [45]. In the case of anionic liposomes, mixtures of 1,2-dioleoyl-sn-glycero3-[phospho-rac-(1-glycerol)] (sodium salt) and 1,2-dioleoyl-sn-glycero-3-phosphoethanolamine as anionic and zwitterionic lipids, respectively, have been used to prepare carriers for the effective ion delivery of plasmid DNA molecules encoding green fluorescence protein [46]. 


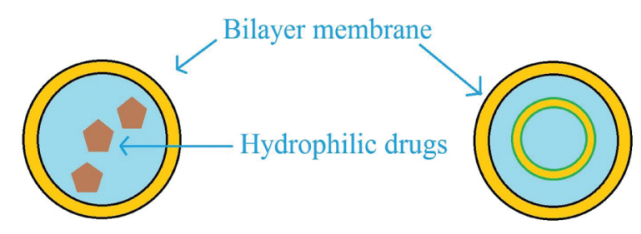

Unilamllar vesicle with a single bilayer membrane

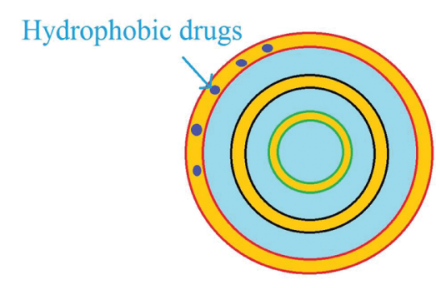

Multilamellar vesicles with two or several bilayer membranes

Figure 3: Unilamellar and multilamellar liposomes with their structures and drug encapsulation approaches [41].

\section{LNPs}

Major disadvantages of liposomes include the lack of affordable preparation methods, low degree of drug loading capacity and stability, and rapid decomposition in the human body before the therapeutic effect can be achieved. Generally, there are four types of LNPs: solid lipid nanoparticles (SLN), nanostructured lipid carrier (NLC), lipid drug conjugate (LDC), and polymer-lipid hybrid nanoparticle (PLN). The first generation of LNPs was introduced in 1993-1996 as SLNs. High stability in the reticuloendothelial system (RES) at the physiologic temperature of $37^{\circ} \mathrm{C}$ and a practically acceptable drug encapsulation could be possible by using solid lipid instead of liquid lipid in the preparation method. These structures have other advantages including an easy large-scale production, simple sterilization, suitable bioavailability, biocompatibility, biodegradability, controlled release of drugs, higher shelf life, efficient drug targeting, and improved drug absorption and dissolution [47]. NLCs are another type of LNPs with both solid and liquid lipids in their composition without perfect crystalline structure. A higher capacity to encapsulate a wide range of drugs with solubility in the liquid and solid phases of lipids is the advantage of these systems over the SLNs [48]. Both SLNs and LNPs have limited capabilities with respect to loading of hydrophilic drugs. In this way, conjugation of hydrophilic drugs with hydrophobic molecules by covalent bonds and salt formation resulted in a new nanostructure named LDC. LDCs can be used for drugs with sensitivity to the acidic conditions of the stomach. For even better loading of hydrophilic drugs, PLNs were introduced as a linkage between ionic polymers and hydrophilic drugs such as gemcitabine [49]. Several polymers such as polycaprolactone and polylactic-coglycolic acid can be utilized for conjugation with drugs in a core-shell structure [50].

\section{Passive targeting}

Liposomes can target cancer tissues by both passive and active targeting strategies (Figure 4). Some mutations can cause uncontrolled division of cells in the body resulting in cancer disease. The basis for the passive targeting of the tumor tissues by liposomes is, mainly, the different pore sizes between the endothelial cells of the tumor microvasculature compared to the 'tighter' structures found in normal capillaries. Therefore, if one prepares liposomes with such a size that allows them to extravasate in the tumor tissues while prohibiting the carriers to exit the capillaries in normal tissues, an ideal targeting goal would be achieved [51], [52], [53]. In addition to the increased permeability, there is a phenomenon in tumor sites commonly known as enhanced permeability and retention effect (EPR). This situation is characterized by the increased blood capillary permeability in the affected tissues with a much lesser return of the fluids to the lymphatic circulation. In this way, the drugs encapsulated in liposomes (up to the size of $400 \mathrm{~nm}$ ) can be accumulated efficiently in tumor sites. Mechanistically, the overexpression of some regulating angiogenesis factors such as vascular endothelial growth factor (VEGF) may result in both chaotic tumor vessel architecture and increased vascular permeability. These factors, ultimately, lead to enhanced permeation and retention [54]. Abraxane ${ }^{\circledR}$ (albumin-bound PTX) is a typical example of a drug delivery system which accumulates in tumors via EPR [55]. 


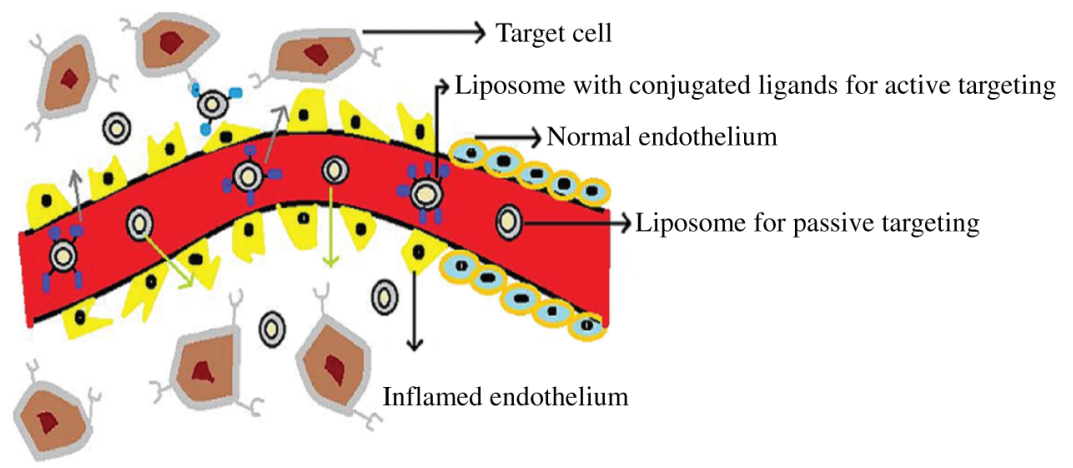

Figure 4: Passive and active targeting of cancer cells for drug targeting by liposomes [51], [52], and [53].

There are several reports of passive targeting by LNPs. For example, sclareol-SLNs with an average particle size of $88 \pm 5 \mathrm{~nm}$ has shown significantly higher growth inhibitor effect on A549 human lung epithelial cancer cells after a period of $48 \mathrm{~h}$ compared to the free drug along with a sustained drug release [56]. Conjugation of curcumin with SLN is another report for passive targeting of tumor tissues used in breast cancer with remarkably higher tissue availability [57]. In a similar study, growth inhibition of Hodgkin's lymphoma xenograft was observed by $50.5 \%$ in the case of curcumin-SLN receiving group [58]. For passive targeting of glioblastoma and melanoma, temozolomide-SLN showed higher inhibition in proliferation of these types of cancer tissues and lower cytotoxicity in healthy cells compared to temozolomide without SLN [59].

\section{Active targeting}

There are several ways to target actively a specific site of body by a drug carrier (Figure 5). In order to achieve the active targeting of cancer sites, a variety of ligands are utilized to exploit any specific antigens expressed by cancer cells. The prostate-specific membrane antigen has been successfully targeted by conjugation of RNA A10 onto PLA-block-PEG co-polymers, which exhibited increased drug delivery to prostate tumor tissue compared to non-targeting nanoparticles [17]. In the case of the active targeting by immunoliposomes, binding to target cells and uptake by the RES are two kinetically competing processes. PEG chains have shown a successful avoiding of the RES uptake of liposomes, thus leading to an elevated blood concentration and enhanced target binding of immunoliposomes. Also, the presence of free PEG did not interfere with the binding of the terminally linked antibody to the antigen in pendant-type immunoliposomes [60], [61].

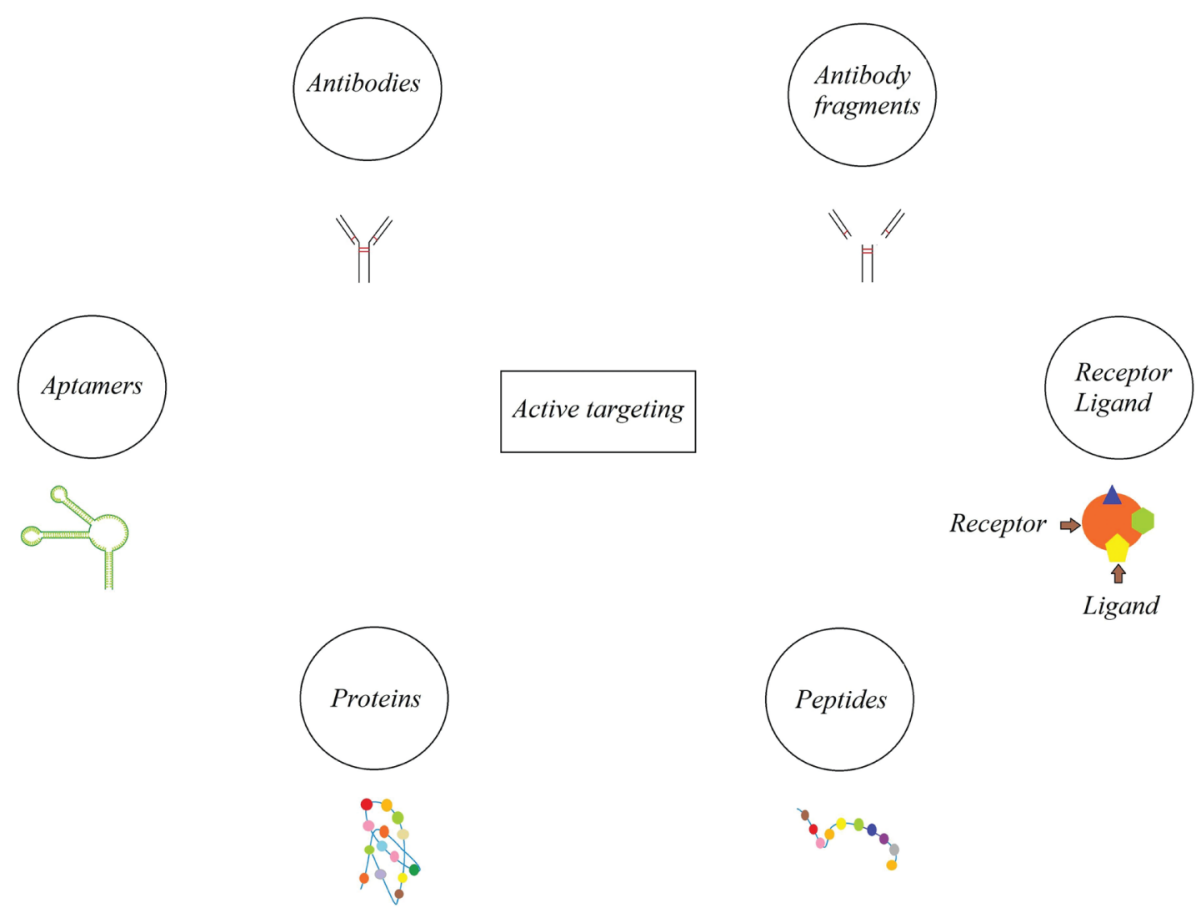

Figure 5: Different approaches of active targeting by liposomes in drug delivery system [17]. 
Breast cancer is characterized by high expression of estrogen receptors including MAPK, HER2/neu, PI3K/Akt, and epidermal growth factor receptor (EGFR)/VEGFR which can be targeted actively by modified liposomes. In this way, the role of IL-6 growth factor is important specifically in complicating the biological situation. For targeting of IL-6, Diacerein encapsulation in Tyr-3-octreotide-PEG-liposomes has shown significant effect on cell division and angiogenesis of breast tumor cells via higher cleavage of caspase 3 and poly ADP ribose polymerase [62].

CD44 is a cell membrane glycoprotein that regulates interaction, adhesion, and migration of cells in the extracellular matrix. These regulations are related to the binding of hyaluronic acid to CD44. In the case of LNPs, hyaluronic acid coated-NLCs loaded by PTX were used to deliver PTX to cancer cells with overexpression of CD44. This nanostructure showed sustained drug release than the free drug, Taxol ${ }^{\circledR}$ [55]. Modification of resveratrol-SLN with Apolipoprotein E demonstrated meaningful permeability through the blood brain barrier hCMEC/D3 cell line with lower cytotoxicity than unmodified resveratrol-SLN [63]. Trans-activating transcriptional activator (TAT) peptide by 86 and 101 amino acids is one of the cell-penetrating peptides that can transfer through the cell membrane without damaging it. In this way, HeLa cells as cervical tumor cells were targeted effectively by TAT-functionalized-SLNs having two antitumor agents including $\alpha$-tocopherol succinate-cisplatin prodrug and PTX [64]. As mentioned, targeting of cancer tissues by antibody is an efficient way of active targeting approach. As a typical example, the receptor for advanced glycation endproducts was used to modify the surface of di-allyl-disulfide-loaded SLN for targeting of triple-negative breast cancer cells [65]. Overexpression of epidermal growth factor receptor variant III (EGFR vIII) in many tumor tissues is another way for targeting. Using anti-EGFR vIII monoclonal antibody conjugated by DSPE-PEG2000-NHS (1,2-distearoylphosphatidylethanolamine-polyethylene glycol 2000-NHS) linker to doxorubicin (Dox)-loaded NLC has shown a meaningful inhibitor effect on the growth of HC2 20d2/c cells [66].

\section{Conclusions}

This review presents recent advances in two drug delivery systems of LNPs and liposomes in passive and active targeting of the cancer sites. Recent surveys have been approved with the great potential for widespread adoption of SLNs in the cancer treatment field. In this way, SLNs are significant candidates for the improvement of drug delivery systems. These structures have major characteristics including suitable biodegradability, higher biocompatibility, lower clearance rates by the RES, the ability for specific targeting of cancer tissues, and sustained controlled-release of drugs. Therefore, it can be concluded that SLNs demonstrate various advantages over conventional chemotherapy and liposomes nanostructures.

Author contributions: All the authors have accepted responsibility for the entire content of this submitted manuscript and approved submission.

Research funding: None declared.

Employment or leadership: None declared.

Honorarium: None declared.

Competing interests: The funding organization(s) played no role in the study design; in the collection, analysis, and interpretation of data; in the writing of the report; or in the decision to submit the report for publication.

\section{References}

[1] Gabizon AA. Pegylated liposomal doxorubicin: metamorphosis of an old drug into a new form of chemotherapy. Cancer Invest 2001;19:424-36.

[2] Gradishar W], Tjulandin S, Davidson N, Shaw H, Desai N, Bhar P, et al. Phase III trial of nanoparticle albumin-bound paclitaxel compared with polyethylated castor oil-based paclitaxel in women with breast cancer. ] Clin Oncol 2005;23:7794-803.

[3] Fu S, Culotta KS, Falchook CS, Hong DS, Myers AL, Zhang YP, et al. Erratum to: Pharmacokinetic evaluation of nanoparticle albuminbound paclitaxel delivered via hepatic arterial infusion in patients with predominantly hepatic metastases. Cancer Chemother Pharmacol 2016;77:881.

[4] Jain MM, Gupte SU, Patil SC, Pathak AB, Deshmukh CD, Bhatt N, et al. Paclitaxel injection concentrate for nanodispersion versus nabpaclitaxel in women with metastatic breast cancer: a multicenter, randomized, comparative phase II/III study. Breast Cancer Res Treat 2016;156:125-34. 
[5] Kapp M, Kosmala A, Kircher S, Luber V, Kunzmann V. Exceptional response to nanoparticle albumin-bound paclitaxel and gemcitabine in a patient with a refractory adenocarcinoma of the ampulla of Vater. Case Rep Oncol 2016;9:15-24

[6] Loong HH, Chan AC, Wong AC. Evolving evidence of the efficacy and safety of nab-paclitaxel in the treatment of cancers with squamous histologies. J Cancer 2016;7:268-75.

[7] Park E, Goldberg NR, Adams S. Nab-paclitaxel-induced cystoid macular edema in a patient with pre-existing optic neuropathy. Anticancer Drugs 2016;27:580-4.

[8] Scheithauer W, Kornek G, Prager G, Stranzl N, Laengle F, Schindl M, et al. Phase II trial of capecitabine plus nab-paclitaxel in patients with metastatic pancreatic adenocarcinoma. ] Gastrointest Oncol 2016;7:234-8.

[9] Chomsky N. What is special about language? SBS Lecture Series: Noam Chomsky: University of Arizona; 2012.

[10] Tinkle S, McNeil SE, Mühlebach S, Bawa R, Borchard G, Barenholz Y, et al. Nanomedicines: addressing the scientific and regulatory gap. Ann N Y Acad Sci 2014;1313:35-56.

[11] Azanza Perea JR, Barberan ]. [Liposomal amphotericin B: a unique pharmacokinetic profile. An unfinished story]. Rev Esp Quimioter 2012;25:17-24.

[12] Perez AP, Altube M], Schilrreff P, Apezteguia G, Celes FS, Zacchino S, et al. Topical amphotericin B in ultradeformable liposomes: formulation, skin penetration study, antifungal and antileishmanial activity in vitro. Colloid Surface B 2016;139:190-8.

[13] Rathore A, Jain A, Gulbake A, Shilpi S, Khare P, Jain A, et al. Mannosylated liposomes bearing Amphotericin B for effective management of visceral leishmaniasis. J Liposome Res 2011;21:333-40.

[14] Skiba-Lahiani M, Hallouard F, Mehenni L, Fessi H, Skiba M. Development and characterization of oral liposomes of vegetal ceramide based amphotericin B having enhanced dry solubility and solubility. Mater Sci Eng C Mater Biol Appl 2015;48:145-9.

[15] Takeda T, Itano H, Kakehashi R, Fukita S, Saitoh M, Takeda S. Direct transbronchial administration of liposomal amphotericin B into a pulmonary aspergilloma. Respir Med Case Rep 2014;11:7-11.

[16] Barenholz Y. Relevancy of drug loading to liposomal formulation therapeutic efficacy.] Liposome Res 2003;13:1-8.

[17] Byrne JD, Betancourt T, Brannon-Peppas L. Active targeting schemes for nanoparticle systems in cancer therapeutics. Adv Drug Deliver Rev 2008;60:1615-26.

[18] Khan DR, Rezler EM, Lauer-Fields ], Fields GB. Effects of drug hydrophobicity on liposomal stability. Chem Biol Drug Des 2008;71:3-7.

[19] Daraee H, Etemadi A, Kouhi M, Alimirzalu S, Akbarzadeh A. Application of liposomes in medicine and drug delivery. Artif Cells Nanomed Biotechnol 2016;44:381-91.

[20] Gasbarri C, Guernelli S, Boncompagni S, Angelini C, Siani G, De Maria P, et al. Fine-tuning of POPC liposomal leakage by the use of betacyclodextrin and several hydrophobic guests. ] Liposome Res 2010;20:202-10.

[21] Laurent S, Vander Elst L, Thirifays C, Muller RN. Relaxivities of paramagnetic liposomes: on the importance of the chain type and the length of the amphiphilic complex. Eur Biophys ] 2008;37:1007-14.

[22] Schwendener RA, Schott H. Liposome formulations of hydrophobic drugs. Methods in molecular biology (Clifton, N]) 2010;605:129-38.

[23] Sikorska E, Ilowska E, Wyrzykowski D, Kwiatkowska A. Membrane structure and interactions of peptide hormones with model lipid bilayers. Biochim Biophys Acta 2012;1818:2982-93.

[24] Takeshita K, Okazaki S, Kansui H. Effect of cholesterol on distribution of stable, hydrophobic perchlorotriphenylmethyl triethylester radical incorporated in lecithin liposomal membranes. Chem Pharm Bull 2011;59:624-8.

[25] Xia S, Tan C, Zhang Y, Abbas S, Feng B, Zhang X, et al. Modulating effect of lipid bilayer-carotenoid interactions on the property of liposome encapsulation. Colloid Surface B 2015;128:172-80.

[26] Nie F, Yu X, Huang M, Wang Y, Xie M, Ma H, et al. Long noncoding RNA ZFAS1 promotes gastric cancer cells proliferation by epigenetically repressing KLF2 and NKD2 expression. Oncotarget 2017;8:38227.

[27] Rosenberg SA, Restifo NP. Adoptive cell transfer as personalized immunotherapy for human cancer. Science (New York, NY) 2015;348:628.

[28] Kasimir-Bauer S, Mayer S, Bojko P, Borquez D, Neumann R, Seeber S. Survival of tumor cells in stem cell preparations and bone marrow of patients with high-risk or metastatic breast cancer after receiving dose-intensive or high-dose chemotherapy. Clin Cancer Res 2001;7:1582-9.

[29] Mellman I, Coukos G, Dranoff G. Cancer immunotherapy comes of age. Nature 2011;480:480

[30] Peccatori FA, Azim Jr HA, Orecchia R, Hoekstra H], Pavlidis N, Kesic V, et al. Cancer, pregnancy and fertility: ESMO Clinical Practice Guidelines for diagnosis, treatment and follow-up. Ann Oncol 2013;24:vi160-vi7o.

[31] Melero I, Gaudernack C, Cerritsen W, Huber C, Parmiani C, Scholl S, et al. Therapeutic vaccines for cancer: an overview of clinical trials. Nat Rev Clin Oncol 2014;11:509.

[32] Demaria M, O'Leary MN, Chang ], Shao L, Liu S, Alimirah F, et al. Cellular senescence promotes adverse effects of chemotherapy and cancer relapse. Cancer Discov 2017;7:165-76.

[33] Ohnishi S, Takeda H. Herbal medicines for the treatment of cancer chemotherapy-induced side effects. Front Pharmacol $2015 ; 6: 14$

[34] Chajar CM. Metastasis prevention by targeting the dormant niche. Nat Rev Cancer 2015;15:238.

[35] Russell S], Peng K-W, Bell JC. Oncolytic virotherapy. Nat Biotechnol 2012;30:658.

[36] Xie ], Codd C, Mo K, He Y. Differential adverse event profiles associated with BCC as a preventive tuberculosis vaccine or therapeutic bladder cancer vaccine identified by comparative ontology-based VAERS and literature meta-analysis. PLoS One 2016;11:e0164792.

[37] Kamta ], Chaar M, Ande A, Altomare DA, Ait-Oudhia S. Advancing cancer therapy with present and emerging immuno-oncology approaches. Front Oncol 2017;7:64.

[38] Sánchez-Paulete AR, Cueto F], Martínez-López M, Labiano S, Morales-Kastresana A, Rodríguez-Ruiz ME, et al. Cancer immunotherapy with immunomodulatory anti-CD137 and anti-PD-1 monoclonal antibodies requires BATF3-dependent dendritic cells. Cancer Discov 2016;6:71.

[39] Jain RK. Delivery of molecular medicine to solid tumors: lessons from in vivo imaging of gene expression and function. ] Control Release 2001;74:7-25 
[40] Akbarzadeh A, Rezaei-Sadabady R, Davaran S, Joo SW, Zarghami N, Hanifehpour Y, et al. Liposome: classification, preparation, and applications. Nanoscale Res Lett 2013;8:102.

[41] Alavi M, Karimi N, Safaei M. Application of various types of liposomes in drug delivery systems. Advanced Pharmaceutical Bulletin. 2017;7:3-9.

[42] Patil YP, Jadhav S. Novel methods for liposome preparation. Chem Phys Lipids 2014;177:8-18.

[43] Luo D, Carter KA, Razi A, Ceng ], Shao S, Giraldo D, et al. Doxorubicin encapsulated in stealth liposomes conferred with light-triggered drug release. Biomaterials 2016;75:193-202.

[44] Anselmo AC, Mitragotri S. Nanoparticles in the clinic. Bioeng Transl Med 2016;1:10-29.

[45] Shim G, Kim M-G, Park JY, Oh Y-K. Application of cationic liposomes for delivery of nucleic acids. Asian ] Pharm Sci 2013;8:72-80.

[46] Patil SD, Rhodes DG, Burgess D]. Anionic liposomal delivery system for DNA transfection. AAPS ] 2004;6:13-22.

[47] Kaur IP, Verma MK. Process for preparing solid lipid sustained release nanoparticles for delivery of vitamins. Google Patents; 2018.

[48] Sadegh MS, Azadi A, Izadi Z, Kurd M, Dara T, Dibaei M, et al. Brain Delivery of Curcumin Using Solid Lipid Nanoparticles and Nanostructured Lipid Carriers: Preparation, Optimization, and Pharmacokinetic Evaluation. ACS Chemical Neuroscience 2018. 10.1021/acschemneuro.8boos10.

[49] Zhao X, Li F, Li Y, Wang H, Ren H, Chen ], et al. Co-delivery of HIF1 $\alpha$ siRNA and gemcitabine via biocompatible lipid-polymer hybrid nanoparticles for effective treatment of pancreatic cancer. Biomaterials 2015;46:13-25.

[50] Wakaskar RR. Ceneral overview of lipid-polymer hybrid nanoparticles, dendrimers, micelles, liposomes, spongosomes and cubosomes. J Drug Target 2018;26:311-8.

[51] Zhang L, Jiang Y, Zheng Y, Zeng Y, Yang Z, Huang G, et al. Selective killing of Burkitt's lymphoma cells by mBAFF-targeted delivery of PinX1. Leukemia 2011;25:331-40.

[52] Ravar F, Saadat E, Cholami M, Dehghankelishadi P, Mahdavi M, Azami S, et al. Hyaluronic acid-coated liposomes for targeted delivery of paclitaxel, in-vitro characterization and in-vivo evaluation. J Control Release 2016;229:10-22.

[53] Szczepanowicz K, Bzowska M, Kruk T, Karabasz A, Bereta ], Warszynski P. Pegylated polyelectrolyte nanoparticles containing paclitaxel as a promising candidate for drug carriers for passive targeting. Colloid Surface B 2016;143:463-71.

[54] Choi I-K, Strauss R, Richter M, Yun C-O, Lieber A. Strategies to increase drug penetration in solid tumors. Front Oncol $2013 ; 3: 193$.

[55] Yang X-Y, Li Y-X, Li M, Zhang L, Feng L-X, Zhang N. Hyaluronic acid-coated nanostructured lipid carriers for targeting paclitaxel to cancer. Cancer Lett 2013;334:338-45.

[56] Hamishehkar H, Bahadori MB, Vandghanooni S, Eskandani M, Nakhlband A, Eskandani M. Preparation, characterization and antiproliferative effects of sclareol-loaded solid lipid nanoparticles on A549 human lung epithelial cancer cells. J Drug Deliv Sci Tec 2018;45:272-80

[57] Tajbakhsh A, Hasanzadeh M, Rezaee M, Khedri M, Khazaei M, ShahidSales S, et al. Therapeutic potential of novel formulated forms of curcumin in the treatment of breast cancer by the targeting of cellular and physiological dysregulated pathways. J Cell Physiol 2018;233:2183-92.

[58] Guorgui ], Wang R, Mattheolabakis G, Mackenzie CG. Curcumin formulated in solid lipid nanoparticles has enhanced efficacy in Hodgkin's lymphoma in mice. Arch Biochem Biophys 2018;648:12-9.

[59] Clemente N, Ferrara B, Gigliotti LC, Boggio E, Capucchio TM, Biasibetti E, et al. Solid lipid nanoparticles carrying temozolomide for melanoma treatment. Preliminary in vitro and in vivo studies. Int ] Mol Sci 2018;19:255.

[60] Harashima H, Kiwada H. The pharmacokinetics of liposomes in tumor targeting. Adv Drug Deliver Rev 1999;40:1-2.

[61] Düzgüneş N, Nir S. Mechanisms and kinetics of liposome-cell interactions. Adv Drug Deliver Rev 1999;40:3-18.

[62] Bharti R, Dey G, Banerjee I, Dey KK, Parida S, Kumar BNP, et al. Somatostatin receptor targeted liposomes with Diacerein inhibit IL-6 for breast cancer therapy. Cancer Lett 2017;388:292-302.

[63] Neves AR, Queiroz JF, Reis S. Brain-targeted delivery of resveratrol using solid lipid nanoparticles functionalized with apolipoprotein E. ] Nanobiotechnol 2016;14:27.

[64] Liu B, Han L, Liu ], Han S, Chen Z, Jiang L. Co-delivery of paclitaxel and TOS-cisplatin via TAT-targeted solid lipid nanoparticles with synergistic antitumor activity against cervical cancer. Int] Nanomed 2017;12:955-68.

[65] Siddhartha VT, Pindiprolu SK, Chintamaneni PK, Tummala S, Nandha Kumar S. RACE receptor targeted bioconjuguate lipid nanoparticles of diallyl disulfide for improved apoptotic activity in triple negative breast cancer: in vitro studies. Artif Cells Nanomed Biotechnol 2018;46:387-97.

[66] Abdolahpour S, Toliyat T, Omidfar K, Modjtahedi H, Wong A], Rasaee M], et al. Targeted delivery of doxorubicin into tumor cells by nanostructured lipid carriers conjugated to anti-ECFRvIII monoclonal antibody. Artif Cells Nanomed Biotechnol 2018;46:89-94. 